09

\title{
Многомерные предельно короткие оптические импульсы в силицене
}

\author{
(C) Н.Н. Конобеева, ${ }^{1}$ М.Б. Белоненко ${ }^{1,2}$ \\ ${ }^{1}$ Волгоградский государственный университет \\ ${ }^{2}$ Волгоградский институт бизнеса \\ E-mail: yana_nn@inbox.ru
}

Поступило в Редакцию 28 апреля 2016 г.

Изучена эволюция двумерных и трехмерных предельно коротких оптических импульсов в силицене. Получено эффективное уравнение, которое имеет вид нелинейного волнового уравнения с насыщающейся нелинейностью. Исследованы импульсы, представляющие собой электромагнитное поле, состоящее из одного и двух колебаний.

DOI: $10.21883 /$ PJTF.2017.08.44534.16316

В последние годы наблюдается повышенное внимание исследователей к нелинейному распространению света в графеноподобных структурах, имеющих уникальные электрофизические свойства, ценные в области современной микро- и наноэлектроники [1]. К таким материалам относится, например, силицен, состоящий из одного слоя атомов кремния в гексагональной решетке [2,3]. Отличительной особенностью силицена можно назвать более сильное спин-орбитальное взаимодействие по сравнению с графеном. Одним из наиболее интересных предсказаний для него является возникновение запрещенной зоны, что может повлечь за собой возникновение перехода между зонным и топологическим изолятором. При этом нельзя оставить без внимания то обстоятельство, что кремний до сих пор остается основным элементом устройств современной микроэлектроники.

В недавних работах авторами изучался вопрос распространения одномерных предельно коротких электромагнитных импульсов в силиценовых волноводах [4]. Было показано, что начиная с некоторого момента времени наблюдается инверсия сигнала, при этом амплитуда инвертированного сигнала практически в два раза превосходит амплитуду первоначального. Таким образом, можно говорить об усилении 
предельно коротких импульсов при резком изменении их формы. При этом остался нерешенным ряд вопросов, связанных с изучением распространения многомерных (2D и 3D) импульсов в силицене. Опираясь на вышеизложенные обстоятельства, представляется важным исследовать взаимодействие таких импульсов с силиценом, где ожидаются новые эффекты с широким диапазоном практических приложений.

В длинноволновом приближении гамильтониан для силицена можно записать в виде $[5,6]$

$$
H=v\left(\xi k_{x} \sigma_{x}+k_{y} \sigma_{y}\right)-0.5 \xi \Delta_{S O} \tau_{z} \sigma_{z}+0.5 \Delta_{z} \sigma_{z},
$$

где $\xi- \pm$ знак долины для двух дираковских точек, $v-$ скорость дираковских электронов, $\mathbf{p}=\left(k_{x}, k_{y}\right)$ - квазиимпульс электронов, $\Delta_{S O}-$ величина спин-орбитальной щели для силицена, $\Delta z$ - потенциал на одном узле решетки, причем $\Delta z=E_{z} d, E_{z}$ - постоянное электрическое поле, $d$ - расстояние между двумя подрешеточными плоскостями, $\sigma_{i}, \tau_{i}$ - матрицы Паули. Типичные значения параметров гамильтониана силицена [7]:

$$
v=5.5 \cdot 10^{5} \mathrm{~m} / \mathrm{s}, \quad \Delta_{S O}=3.9 \cdot 10^{5} \mathrm{meV} .
$$

Записывая гамильтониан в матричной форме, получаем собственные числа:

$$
\varepsilon_{\sigma \xi}= \pm \sqrt{v^{2} k^{2}+\frac{1}{4}\left(\Delta_{z}-\sigma \xi \Delta_{S O}\right)^{2}}
$$

где $\sigma$ - спин электрона (спин „вверх““ и „вниз““).

Проводя аналогичные рассуждения [4], можно записать уравнение на распространение предельно короткого импульса

$$
\frac{\partial^{2} \mathbf{A}}{\partial x^{2}}+\frac{\partial^{2} \mathbf{A}}{\partial z^{2}}-\frac{\partial^{2} \mathbf{A}}{\partial t^{2}}+4 \pi \Phi(\mathbf{A})=0,
$$

здесь скорость света $c=1, \Phi(\mathbf{A})$ определяется при нахождении плотности электрического тока $j$ :

$$
\mathbf{j}=e \int_{-\Delta}^{\Delta} \int_{-\Delta}^{\Delta} d k_{x} d k_{y} v_{y}\left(\mathbf{k}-\frac{e}{c} \mathbf{A}(x, t)\right),
$$

где $v_{y}(\mathbf{k})=\frac{\partial \varepsilon\left(k_{x}, k_{y}\right)}{\partial k_{y}}$, электрическое поле $E$ направлено вдоль оси $y$ и рассматривается в калибровке $\mathbf{E}=-\partial \mathbf{A} / c \partial t$.

4 Письма в ЖТФ, 2017, том 43, вып. 8 
Область интегрирования по импульсам в (4) можно определить из условия равенства числа частиц

$$
\int_{-\Delta}^{\Delta} \int_{-\Delta}^{\Delta} d k_{x} d k_{y}=\int_{Z B} \int d k_{x} d k_{y}\left\langle a_{k x, k y}^{+} a_{k x, k y}\right\rangle,
$$

$a_{k x, k y}^{+} a_{k x, k y}-$ операторы рождения/уничтожения электронов с квазиимпульсом $k$, интегрирование справа ведется по зоне Бриллюэна.

В трехмерном случае уравнение (3) в цилиндрической системе координат примет следующий вид:

$$
\mathbf{A}_{t t}=\frac{1}{r} \frac{\partial}{\partial r}\left(r \frac{\partial \mathbf{A}}{\partial r}\right)+\frac{\partial^{2} \mathbf{A}}{\partial z^{2}}+\frac{1}{r^{2}} \frac{\partial^{2} \mathbf{A}}{\partial \phi^{2}}+4 \pi \Phi(\mathbf{A})
$$

Далее везде полагаем, что в силу цилиндрической симметрии $\frac{\partial}{\partial \varphi} \rightarrow 0$. В силу неоднородности поля вдоль некоторой оси, ток также неоднороден, вследствие чего происходит накопление заряда в какой-то области. Из закона сохранения заряда можно заключить, что существенное влияние на накопленный заряд оказывает длительность предельно короткого импульса. Сделанные авторами оценки (накопленный заряд составляет примерно 1-2\% от заряда, дающего вклад в ток) позволяют полагать, что эффектом накопления заряда для фемтосекундных импульсов можно пренебречь [8].

Исследуемые уравнения (3) и (5) решались численно [9]. Начальное условие в двумерном случае выбиралось в виде предельно короткого импульса, состоящего из одного колебания $(n=0)$ и двух колебаний $(n=1)$ и может быть записано в следующем виде:

$$
\begin{gathered}
A(x, z, 0)=Q z^{n} \exp \left(-z^{2} / \gamma_{z}^{2}\right) \exp \left(-x^{2} / \gamma_{x}^{2}\right), \\
\frac{d A(x, z, 0)}{d t}=\frac{2 z^{n+1} v_{z}}{\gamma_{z}^{2}} Q \exp \left(-z^{2} / \gamma_{z}^{2}\right) \exp \left(-x^{2} / \gamma_{x}^{2}\right),
\end{gathered}
$$

здесь $Q-$ амплитуда импульса, $v_{z}$ - его начальная скорость в направлении $z, \gamma_{z, x}$ определяют ширину импульса. В качестве эволюционной координаты выбрано время.

Начальное условие в трехмерном случае также выбиралось в двух видах - импульса, состоящего из одного колебания $(n=0)$ и двух

Письма в ЖТФ, 2017, том 43, вып. 8 
колебаний $(n=1)$ :

$$
\begin{gathered}
A(z, r, 0)=Q\left(z-z_{0}\right)^{n} \exp \left(-\frac{\left(z-z_{0}\right)^{2}}{\gamma_{z}^{2}}\right) \exp \left(-\frac{r^{2}}{\gamma_{r}^{2}}\right), \\
\frac{d A(z, r, 0)}{d t}=2 Q \frac{\left(z-z_{0}\right)^{n+1} v_{z}}{\gamma_{z}^{2}} \exp \left(-\frac{\left(z-z_{0}\right)^{2}}{\gamma_{z}^{2}}\right) \exp \left(-\frac{r^{2}}{\gamma_{r}^{2}}\right),
\end{gathered}
$$

где $r-$ радиус, $Q-$ амплитуда, $\gamma_{z}, \gamma_{r}$ определяют ширину импульса, $z_{0}$ - начальное смещение центра импульса. Данное начальное условие соответствует тому, что на образец подается предельно короткий импульс, состоящий из одного колебания электрического поля. Значения энергетических параметров выражались в единицах $\Delta$. Отметим, что, как и в 2D-случае, эволюционной переменной является время.

Возникающая эволюция двумерного электромагнитного поля при его распространении по образцу представлена на рис. 1.

Количество колебаний электрического поля оказывает существенное влияние на форму предельно короткого оптического импульса. Видно, что в случае одного колебания поля импульс распространяется более устойчиво, без появления за ним каких-либо „хвостов“. Такое поведение, с одной стороны, обусловлено дисперсией, которая приводит к уширению оптического импульса, а с другой - связано с нелинейностью уравнения (3), которая определяет его „сужение“. Таким образом, стабильное распространение импульса возможно благодаря балансу между этими двумя процессами в силицене.

Возникающая эволюция трехмерного электромагнитного поля при его распространении по образцу представлена на рис. 2.

Результаты аналогичны двумерному случаю. В случае импульса нулевой площади за основным импульсом появляется „хвост“, что говорит в пользу электромагнитного поля с одним колебанием для обеспечения устойчивости его распространения по образцу.

Стоит отметить, что импульс с одним колебанием поля, в силу того, что его спектр доходит и до нулевых частот, может быть использован при генерации терагерцовых импульсов. А импульс с двумя колебаниями поля может быть использован в спектроскопии быстро протекающих процессов.

Отметим, что, хотя импульсы и испытывают некое уширение, связанное с дисперсией, основная часть энергии импульса остается сосредоточенной в ограниченной области пространства, и в этом

$4^{*}$ Письма в ЖТФ, 2017, том 43, вып. 8 

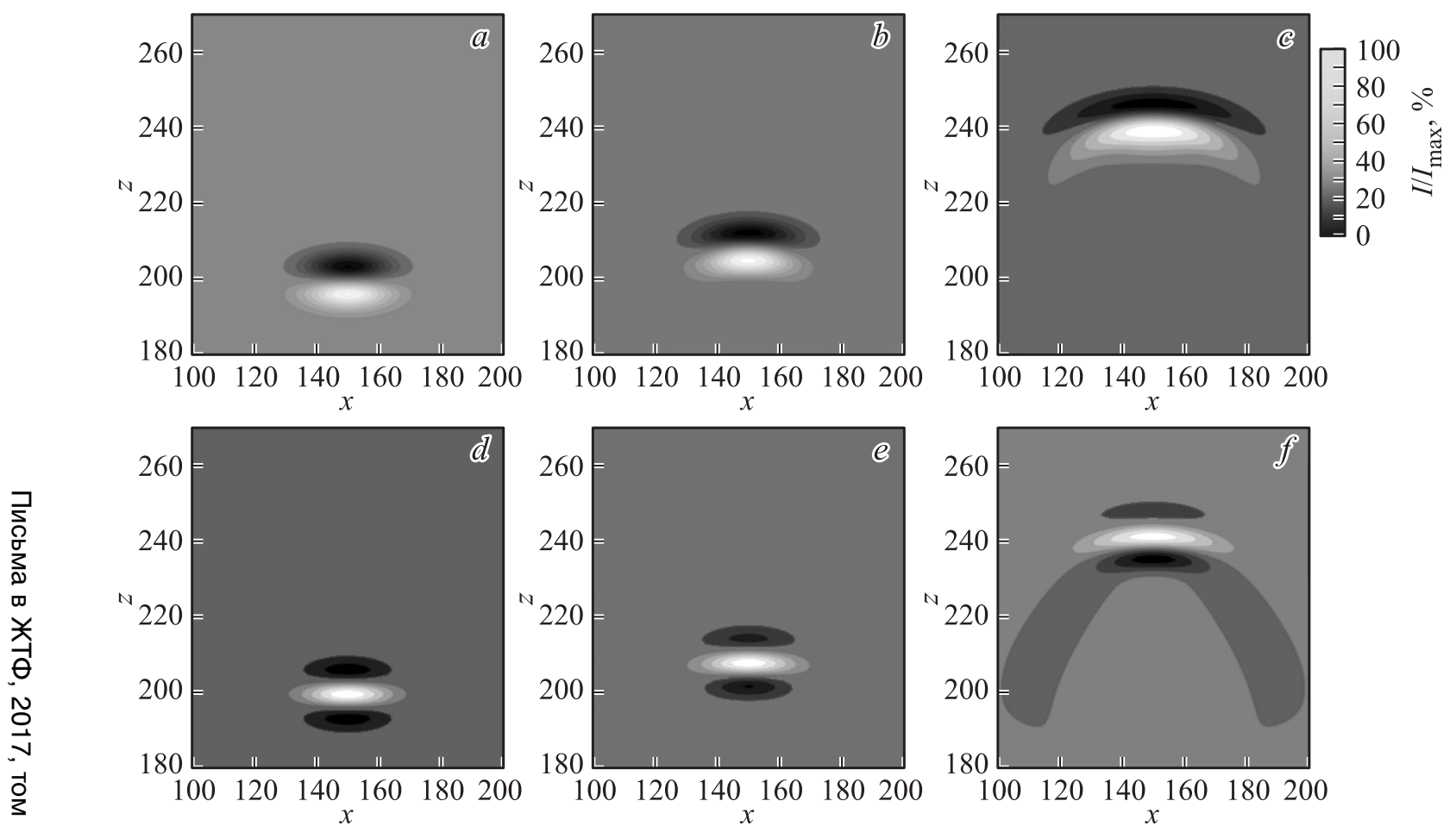

Рис. 1. Интенсивность двумерного электромагнитного импульса $I(x, z, t)=E^{2}(x, z, t)$, состоящего из одного $(a, b, c)$ и двух колебаний $(d, e, f)$ электрического поля в различные моменты времени: $a, d-$ исходная форма импульса; $b, e-t=1.0 \cdot 10^{-13} \mathrm{~s} ; c, f-t=5.0 \cdot 10^{-13} \mathrm{~s}$. Единица по оси $x$ соответствует $30 \mathrm{~nm}$, по оси $z-20 \mathrm{~nm}$. 

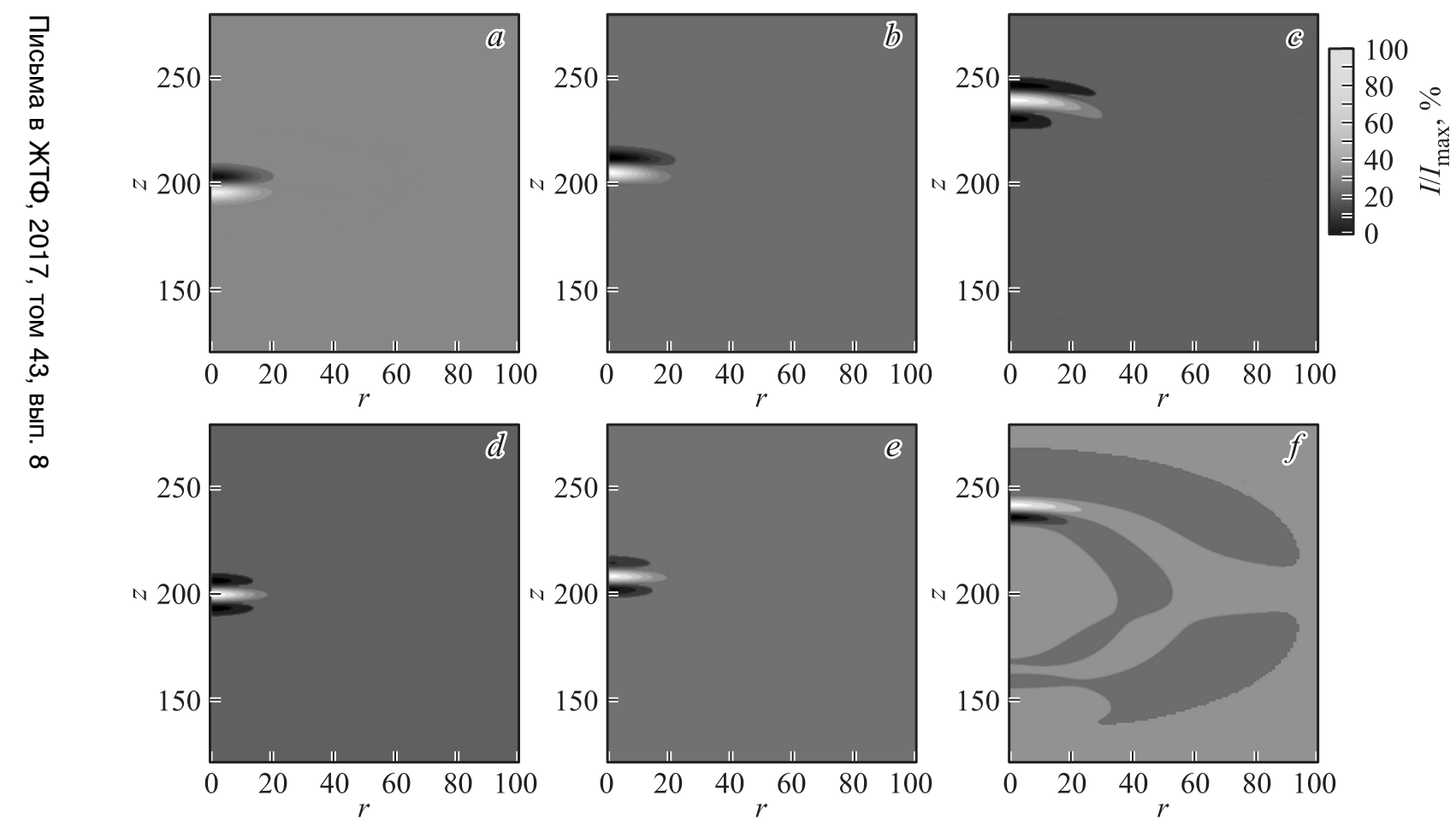

Рис. 2. Интенсивность трехмерного электромагнитного импульса $I(r, z, t)=E^{2}(r, z, t)$, состоящего из одного $(a, b, c)$ и двух колебаний $(d, e, f)$ электрического поля в различные моменты времени: $a, d-$ исходная форма импульса; $b, e-t=1.0 \cdot 10^{-13} \mathrm{~s} ; c, f-t=5.0 \cdot 10^{-13} \mathrm{~s}$. Единица по оси $r$ соответствует $30 \mathrm{~nm}$, по оси $z-20 \mathrm{~nm}$. 
смысле распространение импульса устойчиво. Обратим внимание и на то обстоятельство, что при распространении импульсов не происходит излучение волн, что также свидетельствует в пользу их устойчивости.

В результате проведенного исследования можно сделать следующие выводы:

1. Получено эффективное уравнение для описания динамики многомерных предельно коротких оптических импульсов в силицене.

2. Предельно короткий оптический импульс распространяется с незатухающей амплитудой, что является следствием баланса дисперсии и нелинейности системы.

3. Предельно короткий оптический импульс, состоящий из двух колебаний электрического поля, при своем распространении вызывает появление за собой „хвоста“, что можно связать с возбуждением импульсом нелинейных волн.

4. Показана возможность стабильного распространения предельно коротких импульсов в силицене, что важно для практических приложений в микроэлектронике на основе кремния.

Работа поддержана Министерством образования и науки РФ (проект № MK-4562.2016.2), а также Российским фондом фундаментальных исследований (грант № 16-07-01265 А).

\section{Список литературы}

[1] Novoselov K.S., Jiang D., Schedin F. et al. // Proc. Nat. Acad. Sci. USA. 2005. V. 102. P. 10451.

[2] Aufray B., Kara A., Oughaddou H. et al. // Appl. Phys. Lett. 2010. V. 96. P. 183102.

[3] Padova P., Quaresima C., Ottaviani C. et al. // Appl. Phys. Lett. 2010. V. 96. P. 261905.

[4] Конобеева Н.Н., Белоненко М.Б. // Письма в ЖТФ. 2013. Т. 39. В. 12. C. 87-94.

[5] Ezawa M. // New J. Phys. 2012. V. 14. P. 033003.

[6] Ezawa M. // Phys. Rev. Lett. 2012. V. 109. P. 055502.

[7] Ezawa M. // Phys. Soc. Jpn. 2015. V. 84. P. 121003.

[8] Zhukov A.V., Bouffanais R., Fedorov E.G., Belonenko M.B. // J. Appl. Phys. 2013. V. 114. P. 143106.

[9] Бахвалов Н.С. Численные методы (анализ, алгебра, обыкновенные дифференциальные уравнения). М.: Наука, 1975. 632 с.

Письма в ЖТФ, 2017, том 43, вып. 8 